04

\title{
Метастабильные состояния пылевой плазмы. Сравнение с экспериментом
}

\author{
(C) А.В. Шавлов, ${ }^{1,2}$ В.А. Джуманджи, ${ }^{3}$ А.А. Яковенко ${ }^{1}$ \\ ${ }^{1}$ Институт криосферы Земли ТюмНЦ СО РАН, \\ 625000 Тюмень, Россия \\ 2 Тюменский индустриальный университет, \\ 625000 Тюмень, Россия \\ ${ }^{3}$ Тюменский государственный университет, \\ 620003 Тюмень, Россия \\ e-mail: shavlov@ikz.ru
}

Поступило в Редакцию 8 декабря 2020 г.

В окончательной редакции 27 января 2021 г.

Принято к публикации 1 февраля 2021 г.

С помощью уравнения Пуассона-Больцмана численно определены электростатические потенциалы и вычислены свободные энергии заряженных частиц в равновесной двухтемпературной модели пылевой плазмы. Установлено, что свободные энергии пылевых частиц, ионов и одновременно всех заряженных частиц имеют локальные минимумы (метастабильные состояния) при определенных концентрациях, температуре и заряде частиц. Возможность существования метастабильного состояния частиц в реальной пылевой плазме подтверждена взятыми из литературы экспериментальными данными по пылевым кристаллам и капельным кластерам. Указана возможная связь метастабильных состояний с явлением коагуляции пылевых частиц, а также с образованием пылевых кристаллов.

Ключевые слова: плазма, пылевой кристалл, капельный кластер, коагуляция, уравнение Пуассона-Больцмана, метод Рунге-Кутта, свободная энергия, метастабильное состояние.

DOI: 10.21883/JTF.2021.07.50951.338-20

\section{Введение}

В современном производстве микроэлектроники широко используются плазмохимические методы формирования микропроцессорных кристаллов и травления печатных плат. Одной из основных проблем производства является высокий процент выбраковки образцов из-за неуправляемого зарождения и роста диэлектрических пылевых частиц, загрязняющих плазму в технологическом процессе травления. Пылевые частицы, как правило, несут большой отрицательный заряд до $10^{3}-10^{4}$ единиц элементарного заряда. Установлено, что одним из этапов быстрого увеличения размеров пылевых частиц является этап спонтанной коагуляции [1-10]. Почему могут коагулировать частицы при большом одноименном заряде, является в настоящее время до конца не решенным вопросом физики плазмы. Помимо участия в спонтанной коагуляции, пылевые частицы при определенной концентрации, температуре и заряде способны формировать в плазме пространственно упорядоченные структуры с межчастичным расстоянием, в несколько раз превышающим радиус частиц [11-29]. Подобные структуры - капельные кластеры - образуются также в плазме водных аэрозолей [30]. Можно предположить, что между одноименно заряженными частицами в плазме существуют особые силы притяжения, приводящие к коагуляции или к упорядочению.

В литературе обсуждались такие механизмы, как притяжение за счет бомбардировки частиц разогретыми нейтралами [15], теневое притяжение частиц благодаря затенению частицей потока плазмы на поверхность другой частицы $[15,31]$, кильватерное притяжение из-за притяжения частицы к противоположно заряженному кильватерному следу другой частицы [32]. В работах $[33,34]$ рассматривался механизм электростатического притяжения частиц, вызванный корреляцией электронов и ионов в пространстве между пылевыми частицами в идеальной пылевой плазме.

Настоящая работа посвящена моделированию электростатического взаимодействия частиц в неидеальной неизотермической пылевой плазме и изучению возможности возникновения в ней метастабильных состояний. Для этого рассчитывали электростатические потенциалы частиц, вычисляли и анализировали на наличие локальных минимумов их свободные энергии. Расчеты проводили численным методом Рунге-Кутта на основе системы обыкновенных дифференциальных уравнений, эквивалентной нелинейному уравнению Пуассона-Больцмана. Результаты расчетов сравнили с экспериментальными данными по пылевым кристаллам и капельным кластерам.

\section{1. Формулировка и решение задачи}

Пылевые частицы в плазме заряжаются различными способами в зависимости от типа плазмы (газоразрядная, термическая). В газоразрядной плазме частицы, 
как правило, заряжаются отрицательно из-за того, что электроны гораздо подвижнее ионов, и их поток на частицу больше ионного. В термической плазме частицы могут быть как отрицательно заряженными изза потока электронов на частицы, так и положительно заряженными вследствие термоэлектронной эмиссии с поверхности частиц. В целом пылевая плазма является открытой неравновесной системой, в которой распределение частиц по энергиям скорее определяется равенством потоков ионов и электронов на частицу, чем больцмановскими факторами в случае равновесия. Тем не менее именно идея равновесия лежит в основе изучения газоразрядной и термической плазмы.

В настоящей работе мы будем полагать пылевую плазму неизотермической, состоящей из частиц трех сортов, сильно различающих по массе - макроскопических пылевых частиц, ионов и электронов. При этом считаем, что у каждого сорта частиц вследствие столкновений устанавливается своя равновесная температура. Обмен энергией между частицами разного сорта затруднен из-за большого различия масс частиц. Распределения частиц по энергиям описываются статистикой Больцмана с соответствующей равновесной температурой для каждого сорта. Считаем, что столкновения частиц не слишком часты, чтобы газ частиц в исследуемом объеме метрического пространства можно было рассматривать как идеальный. Для этого необходимо, чтобы длина свободного пробега частиц была не меньше поперечного размера данного объема. Обозначим радиус, концентрацию и температуру пылевых частиц с зарядом в единицах элементарного заряда $Z \gg 1$ (для определенности заряд отрицательный) $R_{z}, N_{z}, T_{z}$; для положительных (однозарядных) ионов соответственно $-R_{i}, N_{i}, T_{i}$; для электронов - $R_{e}, N_{e}, T_{e}\left(T_{z} \gg R_{i}, R_{e}, T_{z} \neq T_{i} \neq T_{e}\right)$. Считаем, что заряженные частицы плазмы испытывают электростатические взаимодействия друг с другом. Предложенная модель плазмы является очень идеализированной. Но, как будет показано ниже, она приводит к возможности существования метастабильных состояний как частиц разных сортов, так и плазмы в целом. Положения использованной модели оправданы только потому, что они привели к результатам, согласующимся с экспериментом.

Уравнение электронейтральности в выбранной модели пылевой плазмы имеет вид

$$
N_{i}=Z N_{z}+N_{e},
$$

а в расчете на одну пылевую частицу:

$$
\underbrace{Z(1-\chi)^{-1}}_{3}=\underbrace{Z}_{1}+\underbrace{\chi Z(1-\chi)^{-1}}_{2},
$$

где обозначен цифрой 1 - заряд пылевой частицы, 2 заряд электронов, 3 - заряд ионов; $\chi=N_{e} / N_{i}$ - доля заряда электронов от заряда ионов.

Свободную энергию плазмы вычислим, следуя схеме расчетов Дебая-Хюкеля [35], также подробно изложен- ной в [36]. Свободная энергия плазмы включает свободную энергию идеального газа и электростатическую поправку к ней. Для определения электростатической поправки воспользуемся уравнением Пуассона-Больцмана с граничными условиями для потенциала заряженной частицы, например, пылевой частицы, в электронейтральном сферическом объеме плазмы, радиусом, многократно превышающим радиус Дебая, содержащем множество частиц всех сортов. (Это так называемая дебаевская модель, в отличие от другой популярной ячеечной модели [37], рассматривающей минимальный объем, содержащий одну пылевую частицу и компенсирующие ее ионы и электроны.) Определив потенциал пылевой частицы, вычтем из него чисто кулоновский потенциал самой частицы. Тем самым найдем потенциал, создаваемый плазмой на поверхности частицы. С помощью данного потенциала вычислим электростатическую поправку к свободной энергии. Затем вычислим полную свободную энергию частицы и исследуем ее на наличие минимума. Аналогично вычислим и исследуем на экстремум свободные энергии иона и электрона, а также полную свободную энергию всех носителей заряда плазмы.

\section{1. Свободная энергия пылевой частицы}

На рис. 1. изображен электронейтральный сферический объем радиуса $d$ для вычисления потенциала центральной пылевой частицы. Полагаем, что радиус $d$ многократно превышает радиус Дебая плазмы. Полный заряд плазмы внутри $d$-сферы считаем равным нулю. Число пылевых частиц внутри сферы, включая центральную частицу, обозначим $Q(Q \gg 1)$, число ионов $-Z Q(1-\chi)^{-1}$, электронов $-\chi Z Q(1-\chi)^{-1}$.

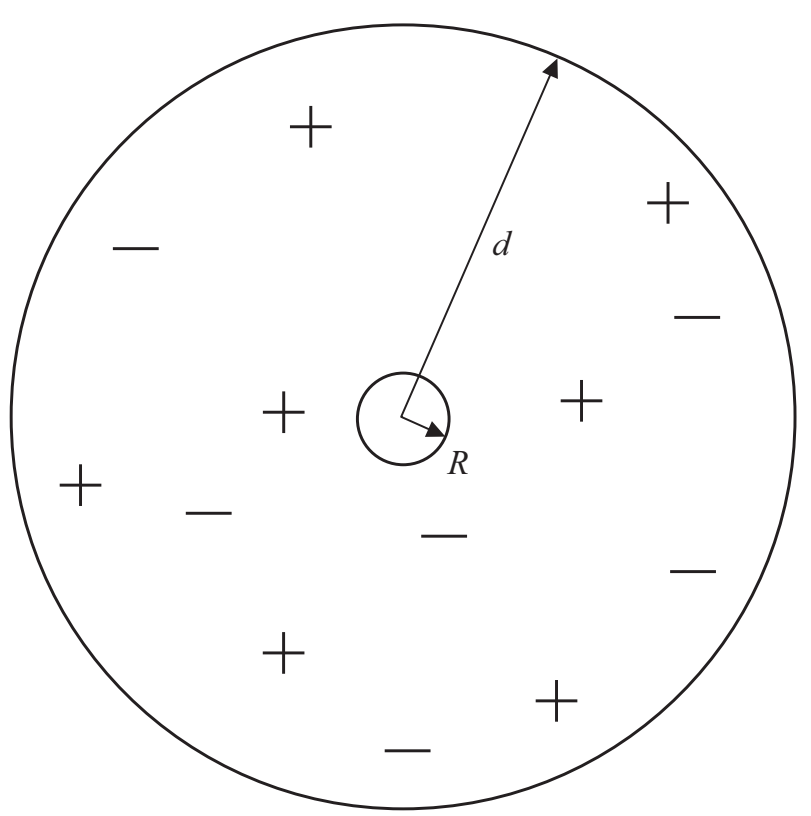

Рис. 1. Сферический электронейтральный объем радиуса $d$ для вычисления потенциала центральной заряженной частицы радиуса $R$. 
Минимальное значение радиуса $d$ при фиксированном значении $Q$ составляет $d_{0}=R_{z}(Q / a)^{1 / 3}(a-$ коэффициент заполнения пространства шарами одинакового радиуса). Оно достигается при плотной упаковке частиц. Например, для гранецентрированной кубической упаковки $a=0.74$. Для того чтобы вычисление потенциала пылевой частицы можно было выполнять с помощью уравнений сплошной среды, будем считать все носители заряда, кроме центральной частицы, равномерно распределенными (размазанными) внутри $d$-сферы, как и их электрический заряд.

Заметим, что время оседлой жизни пылевой частицы в центре $d$-объема определяется периодом пылезвуковых колебаний частицы (точнее, в сфере радиуса Дебая). Оно больше периода электронных плазменных колебаний и периода ионно-звуковых колебаний. Поэтому в уравнении Пуассона-Больцмана следует учитывать корреляцию электронов, ионов и пылевых частиц. Под корреляцией частиц в настоящей работе мы понимаем процесс перераспределения концентрации заряженных частиц в пространстве под действием электрического потенциала центральной частицы $\varphi_{z}$. Корреляция будет учтена, если функции распределения электронов, ионов и пылевых частиц представить в виде распределений Больцмана соответственно $n_{e}=N_{e} \exp \left(\frac{e \varphi_{z}}{k T_{e}}\right), n_{i}=N_{i} \exp \left(\frac{-e \varphi_{z}}{k T_{i}}\right)$, $n_{z}=N_{z} \exp \left(\frac{e Z \varphi_{z}}{k T_{z}}\right)$. Здесь $N_{e}=\frac{Q Z \chi(1-\chi)^{-1}}{V_{z}}$, свободный $N_{i}=\frac{Q Z(1-\chi)^{-1}}{V_{z}}, \quad N_{e}=\frac{Q-1}{V_{z}}, \quad V_{z}=(4 / 3) \pi\left(d^{3}-R_{z}^{3}\right)-$ объем, $k$ - постоянная Больцмана, $e-$ заряд электрона.

Уравнение Пуассона-Больцмана для потенциала пылевой частицы и граничные условия для его производной имеют следующий вид:

$$
\begin{gathered}
\Delta \varphi_{z} \frac{e}{\varepsilon 0}\left(n_{e}-n_{i}+Z n_{z}\right), \\
-\left.\frac{\partial \varphi_{z}}{\partial r}\right|_{R_{z}}=\frac{-Z e}{4 \pi \varepsilon_{0} R_{z}^{2}}, \\
-\left.\frac{\partial \varphi_{z}}{\partial r}\right|_{d}=0,
\end{gathered}
$$

где $\Delta-$ лапласиан в сферических координатах, $\varepsilon_{0}-$ электрическая постоянная.

Первое граничное условие (2a) описывает напряженность электрического поля на поверхности пылевой частицы. Второе граничное условие $(2 \mathrm{~b})$ - напряженность поля на поверхности $d$-объема, равную нулю в силу электронейтральности объема. Заметим, что граничные условия (2a), (2b) определяют значение потенциала с точностью до постоянного слагаемого. Далее в формулах для расчета свободной энергии это постоянное слагаемое потенциала необходимо будет вычесть, т.е. вместо $\varphi_{z}(r)$ будем использовать $\varphi_{z}(r)-\varphi_{z}(d)$.

Задачу решали численно. Для этого уравнение Пуассона-Больцмана свели к эквивалентной системе двух обыкновенных дифференциальных уравнений первого порядка, и решали систему методом Рунге-Кутта четвертого порядка с фиксированным шагом интегрирования, реализованным в функции rkfixed программы Mathcad 11 [38]. Для решения требовались начальные условия для потенциала и его производной на одной из границ, но не значения производной на двух разных границах, как в нашем случае (2a), (2b). Поэтому применяли методику стрельбы. На границе $r=R_{z}$ производную потенциала задавали в соответствии с граничным условием (2a), а величину самого потенциала на данной границе подбирали так, чтобы на другой границе $r=d$ электрическое поле принимало значение, определяемое граничным условием (2b). Заметим, что система уравнений содержит участки быстро и медленно меняющихся решений. Поэтому было удобно перейти к переменному шагу интегрирования по $r$. Для этого в уравнении (1) выполнили замену переменной $r=\exp (y)$, и затем решали уравнение методом Рунге-Кутта с фиксированным шагом интегрирования по переменной $y$. Функция rkfixed возвращала матрицу значений решений системы дифференциальных уравнений, заданных на интервале $\left[\ln \left(R_{z}\right), \ln (d)\right]$. Число строк матрицы результатов задавали равным $10^{4}$.

Из найденного в точке $r=R_{z}$ потенциала далее вычитали потенциал самой пылевой частицы и таким образом определяли потенциал, создаваемый плазмой на поверхности частицы $\varphi P_{z}$ :

$$
\varphi_{P z}=\varphi_{z}\left(R_{z}\right)-\varphi_{z}(d)+\frac{e Z}{4 \pi \varepsilon_{0} R_{z}} .
$$

Электрическая (корреляционная) энергия пылевой частицы равнялась $W=-e Z \varphi_{P z}$. Свободную энергию частицы $F_{z}$ вычисляли по формуле

$$
F_{z}=-k T_{z} \ln V_{z}+T_{z} \int_{T_{z}}^{\infty}\left(\frac{W}{T_{z}^{2}}\right)_{V_{z}} d T_{z},
$$

где первое слагаемое описывает свободную энергию кинетического движения пылевой частицы в идеальном газе, а второе слагаемое описывает электрическую корреляционную поправку к свободной энергии в соответствии с известной термодинамической формулой [36].

\section{2. Свободная энергия иона и электрона}

Для определения свободной энергии иона использовали сферический объем (рис. 1) с ионом, расположенным в его центре. Время оседлой жизни иона в центре шара сравнимо с периодом ионно-звуковых колебаний. Поэтому следует учитывать корреляцию электронов и ионов, а корреляцию пылевых частиц учитывать не нужно, так как для корреляции пылевых частиц недостаточно времени. Исходя из этого, концентрации носителей будем записывать в виде $n_{e}=N_{e} \exp \left(\frac{e \varphi_{i}}{k T_{e}}\right)$, $n_{i}=N_{i} \exp \left(\frac{-e \varphi_{i}}{k T_{i}}\right), \quad n_{z}=N_{z}, \quad$ где $\quad N_{e}=\frac{Q Z \chi(1-\chi)^{-1}}{V_{i}}$, $N_{i}=\frac{Q Z \chi(1-\chi)^{-1}-1}{V_{i}}, N_{Z}=\frac{Q}{V_{i}}, V_{i}=(4 / 3) \pi\left(d^{3}-R_{i}^{3}\right)$. 
Уравнение Пуассона-Больцмана и граничные условия для потенциала иона $\varphi_{i}$ имеют вид, аналогичный (1) $-(2 b)$.

Для определения свободной энергии электрона годится сферический объем (рис. 1) с электроном, расположенным в его центре. Время оседлой жизни электрона в центре шара сравнимо с периодом электронно-звуковых колебаний. Поэтому следует учитывать корреляцию электронов в $d$-объеме, а корреляцию ионов и пылевых частиц учитывать не нужно, так как их корреляция не успевает осуществиться. Исходя из этого, концентрации носителей имеют вид $n_{e}=N_{e} \exp \left(\frac{e \varphi_{e}}{k t_{e}}\right), \quad n_{i}=N_{i}$, $n_{z}=N_{z}, \quad$ где $\quad N_{e}=\frac{Q Z \chi(1-\chi)^{-1}-1}{V_{e}}, \quad N_{i}=\frac{Q Z(1-\chi)^{-1}}{V_{e}}$, $N_{z}=\frac{Q}{V_{e}}, V_{e}=(4 / 3) \pi\left(d^{3}-R_{e}^{3}\right)$.

Свободную энергию всех частиц плазмы в расчете на одну пылевую частицу в соответствии с уравнением электронейтральности вычисляли по формуле

$$
F=F_{z}+Z(1-\chi)^{-1} F_{i}+Z \chi(1-\chi)^{-1} F_{e} .
$$

\section{2. Результаты вычислений и их обсуждение}

На рис. 2 изображены зависимости свободной энергии пылевой частицы, иона, электрона и свободной энергии всех носителей заряда от расстояния между поверхностями пылевых частиц $\delta=2\left(d-d_{0}\right) \sqrt[3]{a / Q}$. Свободная энергия электрона не имеет локальных минимумов и монотонно возрастает при уменьшении расстояния между поверхностями пылевых частиц $\delta$. Свободная энергия иона проходит через максимум и далее понижается с уменьшением расстояния $\delta$ вплоть до нулевого значения. Свободная энергия пылевой частицы, а также свободная энергия одновременно всех носителей заряда проходят

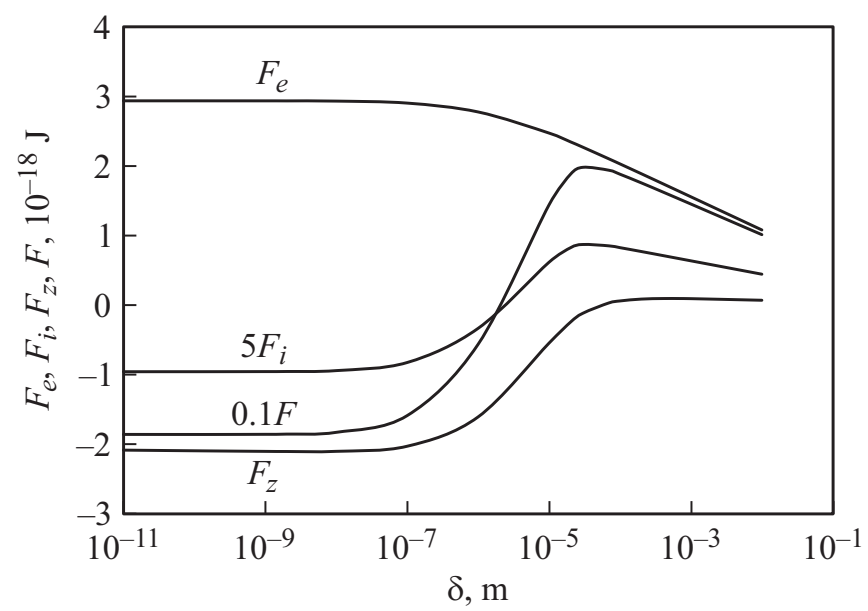

Рис. 2. Свободные энергии пылевой частицы $F_{z}$, иона $F_{i}$, электрона $F_{e}$ и свободная энергия всех носителей заряда $F$ в зависимости от расстояния между поверхностями ближайших пылевых частиц $\delta . T_{e}=5000 \mathrm{~K}, T_{z}=T_{i}=300 \mathrm{~K}, Z=100$, $R=10^{-6} \mathrm{~m}, \chi=0.01$.

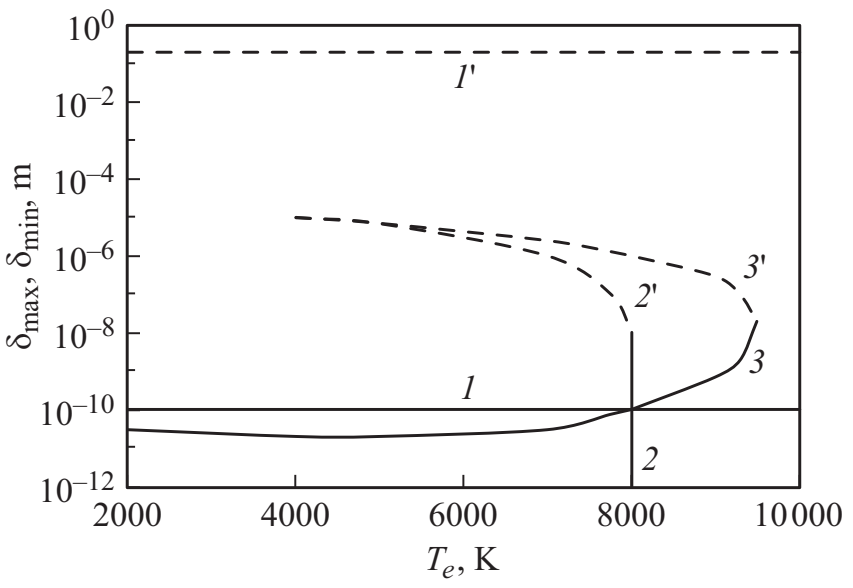

Рис. 3. Расстояния между поверхностями пылевых частиц $\delta_{\max }$ и $\delta_{\min }$ соответственно в точках максимумов и минимумов свободных энергий пылевой частицы (кривые $\left.1^{\prime}, 1\right)$, иона $\left(2^{\prime}, 2\right)$, всех носителей заряда $\left(3^{\prime}, 3\right)$ в зависимости от электронной температуры $T_{e} . Z=500, T_{z}=T_{i}=300 \mathrm{~K}, R=10^{-6} \mathrm{~m}$, $\chi=0.01$.

сначала через максимум, а затем через минимум при уменьшении расстояния $\delta$. Область расстояний с положительным наклоном свободной энергии иона, пылевой частицы и одновременно всех частиц является областью притяжения соответственно отдельных частиц и плазмы в целом. Таким образом, в пылевой плазме могут возникать метастабильные состояния, соответствующие энергетически выгодным фиксированным пространственным положениям частиц. Такие выгодные состояния достигаются благодаря корреляции носителей заряда в плазме. Метастабильные состояния пылевых частиц и ионов могут достигаться при оптимальных параметрах плазмы, обеспечиваемых внешними техническими устройствами. Метастабильное состояние плазмы в целом (состояние из области притяжения одновременно всех частиц плазмы), возможно, способно возникать самопроизвольно даже в тех случаях, когда задаваемые извне параметры плазмы не вполне оптимальны для этого.

На рис. 3 приведены графики расстояний $\delta_{\max }$ и $\delta_{\min }$ между поверхностями пылевых частиц в точках максимумов и минимумов свободных энергий пылевой частицы (кривые $\left.1^{\prime}, 1\right)$, иона $\left(2^{\prime}, 2\right)$, одновременно всех носителей заряда $\left(3^{\prime}, 3\right)$, в зависимости от электронной температуры $T_{e}$. Из рисунка следует, что ширина области притяжения пылевых частиц по шкале $\delta$ (между кривыми $1^{\prime}$ и 1 ) не зависит от электронной температуры. А область притяжения ионов (между кривыми $2^{\prime}$ и 2)) и всех носителей заряда (между кривыми $3^{\prime}$ и 3) существует только при электронных температурах менее $10^{4} \mathrm{~K}$ (при выбранной величине заряда $Z=500$ ).

Проверка зависимости $\delta_{\max }$ и $\delta_{\min }$ от доли заряда электронов $\chi$ показала, что границы областей притяжения пылевых частиц, ионов и плазмы в целом остаются постоянными при изменении $\chi$ в интервале $\chi=\left[10^{-3}, 10^{-1}\right]$. 


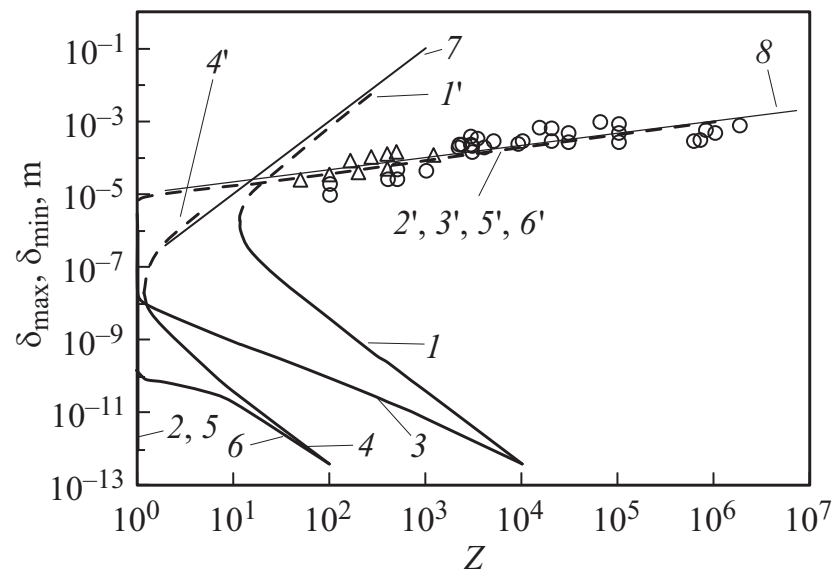

Рис. 4. Расстояния между поверхностями пылевых частиц $\delta_{\max }$ и $\delta_{\min }$ соответственно в точках максимумов и минимумов свободных энергий пылевой частицы (кривые $\left.1^{\prime}, 1\right)$, иона $\left(2^{\prime}, 2\right)$, всех носителей заряда $\left(3^{\prime}, 3\right)$ в зависимости от заряда пылевой частицы $Z$ при ее радиусе $R=10^{-6} \mathrm{~m}$. Кривые $4^{\prime}, 4,5^{\prime}, 5$, $6^{\prime}, 6$ - аналогичные кривые для радиуса пылевой частицы $R=10^{-8} \mathrm{~m} . T_{e}=5000 \mathrm{~K}, T_{z}=T_{i}=300 \mathrm{~K}, \chi=0.01$. Круглые маркеры - экспериментальные значения по пылевой плазме, взятые из литературы. Треугольные маркеры - экспериментальные значения по капельным кластерам. 7, 8 - аналитические кривые.

На рис. 4 приведены графики расстояний $\delta_{\max }$ и $\delta_{\min }$ для свободных энергий пылевой частицы (кривые $1^{\prime}, 1$ ), иона $\left(2^{\prime}, 2\right)$, одновременно всех носителей заряда $\left(3^{\prime}, 3\right)$, в зависимости от величины заряда пылевой частицы $Z$ при радиусе частицы $10^{-6} \mathrm{~m}$. Кривые $\left(4^{\prime}, 4\right),\left(5^{\prime}, 5\right)$, $\left(6^{\prime}, 6\right)$ - аналогичные кривые для радиуса пылевой частицы $R=10^{-8} \mathrm{~m}$. Из рисунка следует, что область притяжения пылевых частиц существует при заряде $Z>1$. Ширина области притяжения возрастает по шкале $\delta$ с увеличением $Z$. Области притяжения ионов и одновременно всех носителей заряда существуют при любом значении заряда $Z$, начиная с единицы и выше. Они также расширяются при увеличении $Z$.

Кривые $1^{\prime}$ и $2^{\prime}$ на рис. 4 удовлетворительно аппроксимируются аналитическими зависимостями 7 и 8 , полученными из условия равенства потенциальной и кинетической энергии, соответственно пылевой частицы и иона. Для пылевой частицы это условие $\frac{Z^{2} e^{2}}{4 \pi \varepsilon_{0}\left(R_{z}+L_{z}\right)}=k T_{z} \quad\left(L_{z}-\right.$ радиус Дебая, определенный по параметрам всех носителей заряда $\left.\left(L_{z} \gg R_{z}\right)\right)$, для иона $\frac{e^{2}}{4 \pi \varepsilon_{0}\left(R_{i}+L_{i}\right)}=k T_{i}\left(L_{i}-\right.$ радиус Дебая, определенный по параметрам ионов и электронов $\left.\left(L_{i} \gg R_{i}\right)\right)$. Из первого условия получаем аналитическую зависимость $\delta=1.5 \cdot 10^{-7} Z^{2}$, которая изображена на рис. 4 , кривая 7. Совпадение с кривой $1^{\prime}$, как отмечали, удовлетворительное. На этой кривой межкапельное расстояние равно примерно одному радиусу Дебая $L_{z}, \delta \approx L_{z}$. Из второго условия следует аналитическая зависимость $\delta=1.5 \cdot 10^{-7} Z^{1 / 3}$, которая проходит существенно ниже, чем кривая $2^{\prime}$. Для совпадения с кривой $2^{\prime}$ нужно предположить, что ион в плазме своим полем в $10^{6}-10^{7}$ раз увеличивает концентрацию электронов вблизи своей поверхности (для этого достаточно электрического потенциала величиной около $5 \mathrm{~V}$, что достигается на расстоянии от поверхности иона, равном радиусу иона). Тогда получим зависимость $\delta=1.5 \cdot 10^{-5} Z^{1 / 3}$ (кривая 8 ), которая удовлетворительно аппроксимирует кривую $2^{\prime}$.

Необходимо отметить особенность поведения кривых $2^{\prime}, 3^{\prime}$ на рис. 3 и кривых $1^{\prime}, 4^{\prime}$ на рис. 4. При попытках расширения расчетных диапазонов в горизонтальном направлении (при $T_{e}<4 \cdot 10^{3}$ для кривых $2^{\prime}, 3^{\prime}$ на рис. 3 и при $Z>30$ и $Z>5$ для кривых $1^{\prime}, 4^{\prime}$ соответственно на рис. 4) данные кривые резко изгибались вверх, стремясь к бесконечным значениям. Объяснение, которое можно предложить в этом случае состоит в том, что используемая в настоящей работе дебаевская модель плазмы с больцмановским распределением частиц начинает приводить к большим ошибкам [37]. Для их уменьшения можно применять какие-либо „кухонные“ методы, например, обрезание потенциала взаимодействия на малых межчастичных расстояниях. Мы подобные методы не использовали, и отмеченное стремление к бесконечности считали недостоверным, и графически не отображали.

Выполненные нами расчеты показали возможность существования метастабильных состояний в равновесной двухтемпературной модели пылевой плазмы. Реальность существования таких состояний в природе необходимо подтверждать экспериментальными данными. Экспериментальные данные для сравнения были взяты из литературы по пылевой плазме и по капельным кластерам в водных аэрозолях. Способы получения плазмы были разные, например, пылевую плазму получали с помощью СВЧ-разряда, прямоточного тлеющего разряда, а также в пламени горения горючих веществ. В большинстве случаев плазма была неизотермической, температура электронов составляла $1-3 \mathrm{eV}$. Капельные кластеры получали в воздухе над поверхностью подогретой воды, а также они самопроизвольно возникали в свободном водяном тумане. Заряд капель приобретался в процессах испарения/конденсации при обмене протонами воды и пара. Плазма водных аэрозолей была изотермической. Из литературы извлекали данные о радиусе частиц, заряде и межчастичном расстоянии (см. таблицу).

Экспериментальные данные из таблицы изображены на рис. 4. Круглыми маркерами представлены данные по пылевой плазме, а треугольными - по капельным кластерам. На рисунке видно, что все экспериментальные точки (круглые и треугольные маркеры) ложатся на кривую $3^{\prime}$, либо в непосредственной близости от нее. Отклонения некоторых точек от положения кривой $3^{\prime}$, на наш взгляд, могут быть связаны как с неточным соответствием выбранной нами модели и реальной плазмы, так и с погрешностью определения плазменных параметров в экспериментах. Положение кривой $3^{\prime}$ не зависит от радиуса пылевых частиц (ср. кривые $3^{\prime}$ 


\begin{tabular}{|c|c|c|c|c|}
\hline Способ получения плазмы & $R, \mu$ & $Z Z$ & $l, \mu$ & Источник \\
\hline СВЧ-разряд & 3.5 & $10^{4}$ & 250 & {$[11]$} \\
\hline СВЧ-разряд & 5 & $3 \cdot 10^{3}$ & 150 & {$[12]$} \\
\hline СВЧ-разряд & 15 & $10^{5}$ & 880 & {$[13]$} \\
\hline Прямоточный тлеющий разряд & 30 & $6 \cdot 10^{5}$ & 300 & {$[14]$} \\
\hline Прямоточный тлеющий разряд & 0.05 & $10^{2}$ & 20 & {$[9]$} \\
\hline Прямоточный тлеющий разряд & 0.01 & $10^{2}$ & 10 & {$[10]$} \\
\hline Плазма планетарных колец & 0.15 & $10^{4}$ & 300 & Обзор $[15]$ \\
\hline Прямоточный тлеющий разряд & 63 & $1.8 \cdot 10^{6}$ & 800 & {$[16]$} \\
\hline Прямоточный тлеющий разряд & 35 & $8 \cdot 10^{5}$ & 600 & {$[16]$} \\
\hline Прямоточный тлеющий разряд & 3 & $2 \cdot 10^{4}$ & 300 & {$[18]$} \\
\hline Термическая плазма в пламени горения & 0.4 & $5 \cdot 10^{2}$ & 27 & {$[17]$} \\
\hline Прямоточный тлеющий разряд & 55 & $10^{6}$ & 500 & {$[17]$} \\
\hline Прямоточный тлеющий разряд & 4 & $10^{5}$ & 500 & {$[17]$} \\
\hline Термическая плазма в пламени горения & 0.5 & $5 \cdot 10^{2}$ & 50 & {$[19]$} \\
\hline Термическая плазма в пламени горения & 0.4 & $5 \cdot 10^{2}$ & 27 & {$[17]$} \\
\hline Прямоточный тлеющий разряд & 55 & $10^{6}$ & 500 & {$[17]$} \\
\hline Прямоточный тлеющий разряд & 4 & $10^{5}$ & 500 & {$[17]$} \\
\hline Прямоточный тлеющий разряд & 10 & $10^{5}$ & 280 & {$[21]$} \\
\hline Прямоточный тлеющий разряд & 2 & $3 \cdot 10^{4}$ & 280 & {$[22]$} \\
\hline Прямоточный тлеющий разряд & 30 & $7 \cdot 10^{5}$ & 320 & {$[22]$} \\
\hline Термическая плазма в пламени горения & 0.4 & $10^{3}$ & 46 & {$[24]$} \\
\hline Прямоточный тлеющий разряд & 5 & $2 \cdot 10^{4}$ & 670 & {$[27]$} \\
\hline Прямоточный тлеющий разряд & 2 & $1.5 \cdot 10^{4}$ & 700 & {$[28]$} \\
\hline СВЧ-разряд & 1 & $5 \cdot 10^{3}$ & 300 & {$[20]$} \\
\hline СВЧ-разряд & 1.7 & $4 \cdot 10^{3}$ & 200 & {$[25]$} \\
\hline Плазма, индуцированная солнечным излучением & 15 & $6.4 \cdot 10^{4}$ & 1000 & {$[26]$} \\
\hline СВЧ-разряд & 1 & $3.4 \cdot 10^{3}$ & 350 & {$[29]$} \\
\hline СВЧ-разряд & 1 & $3 \cdot 10^{3}$ & 220 & {$[29]$} \\
\hline СВЧ-разряд & 1 & $2.9 \cdot 10^{3}$ & 400 & {$[29]$} \\
\hline СВЧ-разряд & 1 & $2.9 \cdot 10^{3}$ & 240 & {$[29]$} \\
\hline СВЧ-разряд & 1 & $2.36 \cdot 10^{3}$ & 240 & {$[29]$} \\
\hline СВЧ-разряд & 1 & $2.16 \cdot 10^{3}$ & 200 & {$[29]$} \\
\hline СВЧ-разряд & 1 & $2.2 \cdot 10^{3}$ & 240 & {$[29]$} \\
\hline Капельный кластер над водой & - & 165 & 84 & {$[39]$} \\
\hline Капельный кластер над водой & - & 272 & 106 & {$[39]$} \\
\hline Капельный кластер над водой & - & 398 & 128 & {$[39]$} \\
\hline Капельный кластер над водой & - & 497 & 146 & [39] \\
\hline Капельный кластер над водой & - & 1207 & 120 & {$[39]$} \\
\hline Капельный кластер в свободном тумане & 7 & 400 & 50 & {$[40]$} \\
\hline Капельный кластер в свободном тумане & 5 & 200 & 40 & {$[40]$} \\
\hline Капельный кластер в свободном тумане & 3 & 100 & 35 & {$[40]$} \\
\hline Капельный кластер в свободном тумане & 2.5 & 50 & 25 & {$[40]$} \\
\hline
\end{tabular}

Примечание. $R$ - радиус пылевой частицы, $Z-$ заряд, $l-$ расстояние между частицами.

и $6^{\prime}$, они совпадают). Данная кривая $3^{\prime}$ соответствует верхней границе удержания одновременно всех частиц плазмы.

Результат удовлетворительного совпадения теоретической кривой $3^{\prime}$ с экспериментальными значениями может служить подтверждением приемлемости выбранной нами равновесной двухтемпературной модели для описания состояний реальной пылевой плазмы. Полученные результаты могут послужить побудительной причиной к анализу возможных механизмов возникновения пылевых кристаллов в плазме и капельных кластеров в водных аэрозолях. Согласно рассмотренной нами модели, суще- ствование пылевых кристаллов и капельных кластеров может быть следствием возникновения метастабильного состояния одновременно всех частиц в плазме или, другими словами, следствием самоудержания плазмы в целом.

Почему экспериментальные точки легли на кривую $3^{\prime}$, а не на кривую 3 , соответсвующую минимальным значениям свободной энергии (нижняя граница области удержания плазмы)? Возможно, это связано с проблемой отвода тепла, выделяющегося при сжатии. Повышение температуры при сжатии может вывести плазму из области удержания, и плазма снова расширится. Проблема 
увеличения температуры, возможно, не возникает при сближении единичных пылевых частиц и они способны достигать состояния, лежащие на кривой 3 .

С помощью теоретических графиков рис. 4 можно предложить физическое объяснение коагуляции частиц в пылевой плазме. Действительно, будем полагать, что коагуляция возможна при расстоянии между пылевыми частицами менее $10^{-10} \mathrm{~m}$, когда вступают в действие межатомные силы. Для частиц малого радиуса, $R=10^{-8} \mathrm{~m}$, нижняя граница области удержания плазмы в целом (кривая 6) располагается по шкале $\delta$ ниже значения $10^{-10} \mathrm{~m}$ при любом $Z \geq 1$. Поэтому мелкие пылевые частицы могут коагулировать. Для частиц большего радиуса, $R=10^{-6} \mathrm{~m}$, коагуляция невозможна при величине заряда частиц $Z<10^{2}$ из-за большого межчастичного расстояния, согласно положению нижней границы области удержания (кривая 3). Для более крупных частиц, например, $R=10^{-4} \mathrm{~m}$, коагуляция невозможна при более высоких значениях заряда, $Z<10^{5}-10^{6}$. Таким образом, крупные частицы с не слишком большим зарядом не коагулируют и могут формировать пространственно упорядоченные структуры пылевые кристаллы - благодаря электростатическому притяжению между частицами в плазме. Мелкие же частицы склонны к коагуляции.

\section{Заключение}

Результаты, полученные в работе, показывают возможность существования метастабильных состояний пылевых частиц, ионов и одновременно всех носителей заряда в равновесной двухтемпературной модели пылевой плазмы. Эти состояния достигаются за счет корреляции носителей заряда, расположенных в пространстве между пылевыми частицами.

Возможность реального существования метастабильного состояния одновременно всех частиц пылевой плазмы подтверждена взятыми из литературы экспериментальными данными по пылевым кристаллам и капельным кластерам. Указана возможная связь метастабильных состояний с явлением коагуляции пылевых частиц.

Сформулированные в работе выводы могут быть полезны для дальнейшего продвижения понимания процессов, протекающих в пылевых кристаллах, коллоидных кристаллах и капельных кластерах. Предложенные представления о механизме коагуляции макрочастиц могут быть полезными в физике облаков и туманов. В данном разделе физической науки процессы коалесценции и роста электрически заряженных капель воды до дождевого размера до сих пор не до конца ясны.

\section{Финансирование работы}

Работа выполнена по государственному заданию ТюмНЦ СО РАН IX.135.2.4.

\section{Конфликт интересов}

Авторы заявляют, что у них нет конфликта интересов.

\section{Список литературы}

[1] Y. Hayashi, K. Tachibana. Jpn. J. Appl. Phys., 33 (1), 4208 (1994).

[2] A. Garscadden. Pure Appl. Chem., 66, 1319 (1994). DOI: $10.1351 /$ pac199466061319

[3] Y. Watanabe, M. Shiratani, H. Kawasaki, S. Singh, T. Fukuzawa, Y. Ueda, H. Ohkura. J. Vac. Sci. Technol. A., 14 (2), 540 (1996). DOI: 10.1116/1.580141

[4] H. Kawasaki, J. Kida, K. Sakamoto, T. Fukuzawa, M. Shiratani, Y. Watanabe. J. Appl. Phys., 83, 5665 (1998). DOI: $10.1063 / 1.367420$

[5] T. Fukuzava, S. Kushima, Y. Matsuoka. J. Appl. Phys., 86, 3543 (1999). DOI: 10.1063/1.371256

[6] A. Bouchoule, L. Boufendi, J. Hermann, A. Plain, T. Hbid, G. Kroesen, W.W. Stoffels. Pure Appl. Chem., 68 (5), 1121 (1996). DOI: 10.1351/pac199668051121

[7] M. Shiratani, S. Maeda, K. Koga, Y. Watanbe. Jpn. J. Appl. Phys., 39 (1), 287 (2000).

[8] М.А. Олеванов, Ю.А. Манкелевич, Т.В. Рахимова. ЖТФ, 73 (10), 51 (2003). [M.A. Olevanov, Yu.A. Mankelevich, T.V. Rahimova. Tech. Phys., 48 (10), 1270 (2003). DOI: $10.1134 / 1.1620120$

[9] K.G. Emeleus, A. Breslin. Int. J. Electron., 29, 1 (1970).

[10] A. Bouchoule, L. Boufendi. Plasma Sources Sci. Technol., 3, 292 (1994).

[11] H. Thomas, G.E. Morfill, V. Demmel, J. Goree, B. Feuerbacher, D. Mohlmann. J. Phys. Rev. Lett., 73, 652 (1994). DOI: 10.1103/PhysRevLett.73.652

[12] J. H. Chu, I. Lin. Physica A, 205, 183 (1994). DOI: $10.1016 / 0378-4371(94) 90498-7$

[13] A. Melzer, T. Trottenberg, A. Piel. Phys. Lett. A, 191, 301 (1994). DOI: 10.1016/0375-9601(94)90144-9

[14] V.E. Fortov, A.F. Nefedov. Phys. Lett. A, 218, 89 (1996). DOI: 10.1016/0375-9601(96)00464-1

[15] В.Н. Цытович. УФН, 167 (1), 57 (1997). DOI: 10.3367/UFNr.0167.199701e.0057

[V.N. Tsytovich. Phys. Usp., 40, 53 (1997). DOI: 10.1070/PU1997v040n01ABEH000201]

[16] А.П. Нефедов, О.С. Ваулина, А.М. Липаев, В.И. Молотков, В.М. Торчинский, В.Е. Фортов, А.В. Чернышев, А.И. Иванов, А.Ю. Калери, Ю.П. Семенов, С.В. Залетин. ЖЭТФ, 122 (4), 778 (2002). [A.P. Nefedov, O.S. Vaulina, O.F. Petrov, V.I. Molotkov, V.M. Torchinskii, V.E. Fortov, A.V. Chernyshev, A.M. Lipaev, A.I. Ivanov, A.Yu. Kaleri, Yu.P. Semenov, S.V. Zaletin. JETP, 95 (4), 673 (2002). DOI: 10.1134/1.1520599]

[17] А.П. Нефедов, О.Ф. Петров, В.Е. Фортов. УФН, 167 (11), 1215 (1997). DOI: 10.3367/UFNr.0167.199711e.1215 [A.P. Nefedov, O.F. Petrov, V.E. Fortov. Phys. Usp., 40, 1163 (1997). DOI: 10.1070/PU1997v040n11ABEH000308]

[18] А.А. Самарян, А.В. Чернышев, О.Ф. Петров, А.П. Нефедов, В.Е. Фортов. ЖЭТФ, 119 (3), 524 (2001).

[A.A. Samaryan, A.V. Chernyshev, O.F. Petrov, A.P. Nefedov, V.E. Fortov. JETP, 92 (3), 454 (2001). DOI: $10.1134 / 1.1364742]$ 
[19] О.С. Ваулина, А.П. Нефедов, А.А. Самарян, О.Ф. Петров, А.В. Чернышев. ЖЭТФ, 117 (3), 537 (2000).

[O.S. Vaulina, A.P. Nefedov, A.A. Samaryan, O.F. Petrov, A.V. Chernyshev. JETP, 90, 470 (2000).

DOI: $10.1134 / 1.559127]$

[20] О.С. Ваулина, А.А. Самарян, Б. Джеймс, О.Ф. Петров, В.Е. Фортов. ЖЭТФ, 123 (6), 1179 (2003).

OO.S. Vaulina, A.A. Samarian, B. James, O.F. Petrov, V.E. Fortov. JETP, 96 (6), 1037 (2003).

DOI: $10.1134 / 1.1591215]$

[21] О.М. Белоцерковский, И.Е. Захаров, А.П. Нефедов, О.А. Синкевич, В.С. Филинов, В.Е. Фортов. ЖЭТФ, 115 (3), 819 (1999).

[O.M. Belotserkovskii, I.E. Zaharov, A.P. Nefedov, O.A. Sinkevich, V.S. Filinov, V.E. Fortov. JETP, 88, 449 (1999). DOI: $10.1134 / 1.558815]$

[22] А.М. Липаев, В.И. Молотков, А.П. Нефедов, О.Ф. Петров, В.М. Торчинский, В.Е. Фортов, А.Г. Храпак, С.А. Храпак. ЖЭТФ, 112 (12), 2030 (1997).

A.M. Lipaev, V.I. Molotkov, A.P. Nefedov, O.F. Petrov, V.M. Torchinskii, V.E. Fortov, A.G. Hrapak, C.F. Hrapak. JETP, 85, 1110 (1997). DOI: 10.1134/1.558383]

[23] В.Е. Фортов, А.Л. Нефедов, О.Ф. Петров, А.А. Самарян, Я.К. Ходатаев, А.В. Чернышев. ЖЭТФ, 116 (11), 1601 (1999). [V.E. Fortov, A.P. Nefedov, O.F. Petrov, A.A. Samaryan, Ya.K. Hodataev, A.V. Chernyshov. JETP, 89 (5), 864 (1999). DOI: 10.1134/1.558926]

[24] В.Е. Фортов, В.С. Филинов, А.Л. Нефедов, О.Ф. Петров, А.А. Самарян, А.М. Липаев. ЖЭТФ, 111 (3), 889 (1997).

[V.E. Fortov, V.S. Filinov, A.P. Nefedov, O.F. Petrov, A.A. Samaryan, A.M. Lipaev. JETP, 84 (3), 489 (1997). DOI: $10.1134 / 1.558167]$

[25] В.Е. Фортов, О.С. Ваулина, О.Ф. Петров, В.И. Молотков, А.В. Чернышев, А.М. Липаев, Г. Морфилл, Х. Томас, Х. Ротермел, С. Храпак, Ю.П. Семенов, А.И. Иванов, С.К. Крикалев, Ю.П. Гидзенко. ЖЭТФ, 123 (4), 798 (2003). [V.E. Fortov, O.S. Vaulina, O.F. Petrov, V.I. Molotkov, A.V. Chernyshev, A.M. Lipaev, G. Morfill, H. Thomas, H. Rotermell, S.A. Khrapak, Yu.P. Semenov , A.I. Ivanov, S.K. Krikalev, Yu.P. Gidzenko. JETP, 96 (4), 704 (2003). DOI: $10.1134 / 1.1574544]$

[26] В.Е. Фортов, А.П. Нефедов, О.С. Ваулина, А.М. Липаев, В.И. Молотков, А.А. Самарян, В.П. Никитский, А.И. Иванов, С.Ф. Савин, А.В. Калмыков, А.Я. Соловьев, П.В. Виноградов. ЖЭТФ, 114 (12), 2004 (1998).

[V.E. Fortov, A.P. Nefedov, O.S. Vaulina, A.M. Lipaev, V.I. Molotkov, A.A. Samaryan, V.P. Nikitskii, A.I. Ivanov, S.F. Savin, A.V. Kalmykov, A.Ya. Solov'ev, P.V. Vinogradov. JETP, 87, 1087 (1998). DOI: 10.1134/1.558598]

[27] О.Ф. Петров, М.М. Васильев, Йе Тун, К.Б. Стаценко, О.С. Ваулина, Е.В. Васильева, В.Е. Фортов. ЖЭТФ, 147 (2), 372 (2015). DOI: 10.7868/S0044451015020169 [O.F. Petrov, M.M. Vasiliev, Ye Tun, K.B. Statsenko, O.S. Vaulina, E.V. Vasilieva, V.E. Fortov. JETP, 120 (3), 327 (2015). DOI: $10.1134 / \mathrm{S} 1063776115020181]$

[28] В.С. Воробьев, О.Ф. Петров, В.Е. Фортов. ЖЭТФ, 131 (4), 750 (2007). [V.S. Vorob'ev, O.F. Petrov, V.E. Fortov. JETP, 104 (4), 661 (2007). DOI: 10.1134/S1063776107040176]

[29] А.В. Зобнин, А.Д. Усачев, О.Ф. Петров, В.Е. Фортов. ЖЭТФ, 122 (3), 500 (2002). [A.V. Zobnin, A.D. Usachev, O.F. Petrov, V.E. Fortov. JETP, 95 (3), 429 (2002). DOI: $10.1134 / 1.1513815]$
[30] А.А. Федорец. Письма в ЖЭТФ, 79 (8), 457 (2004). [A.A. Fedorets. JETP Lett., 79 (8), 372 (2004).

[31] А.М. Игнатов. Физика плазмы, 22, 648 (1996).

[32] M. Nambu, S.V. Vladimirov, P.K. Shukla. Phys. Lett. A, 203, 40 (1995). DOI: 10.1016/0375-9601(95)00380-L

[33] A.V. Shavlov, A.V. Dzhumandzhi. Phys. Lett. A, 377, 3131 (2013). DOI: 10.1016/j.physleta.2013.09.040

[34] А.В. Шавлов, В.А. Джуманджи. ЖТФ, 86 (10), 7 (2016). [A.V. Shavlov, V.A. Dzhumandzhi. Tech. Phys., 16 (10), 1449 (2016). DOI: 10.1134/S1063784216100248]

[35] P. Debye, E. Hiickel. Physik Z., 24, 185 (1923).

[36] Л.Д. Ландау, Е.М. Лифшиц. Теоретическая физика. Статистическая физика (Физматлит, М., 2002), с. 282, т. 5, ч. 1, изд.5-е.

[37] Н.Н. Калиткин, Д.П. Костомаров. Матем. моделирование, 17 (4), 40 (2005).

[38] Mathcad 11. User's Guide. Mathsoft Engineering \& Education. (Inc. Printed in the USA, 2002)

[39] A.V. Shavlov, V.A. Dzhumandzhi, S.N. Romanyuk. Phys. Lett. A, 376 (28-29), 2049 (2012). DOI: $10.1016 /$ j.physleta.2012.05.012

[40] А.В. Шавлов, И.В. Соколов, В.А. Джуманджи. ДАН, 470 (1), 32 (2016). DOI: 10.7868/S086956521621012X [A.V. Shavlov, I.V. Sokolov, V.A. Dzhumandzhi. Dokl. Phys., 61 (9), 429 (2016). DOI: 10.1134/S1028335816070132] 